\title{
Chemical and Enzymatic Probes for Nucleic Acid Structure
}

\section{INTRODUCTION}

O ver the past 10 years, significant progress has been made in elucidating the structure of nucleic acids to atomic resolution. By comparison with proteins, however, relatively few high-resolution nucleic structures have been determined. One main reason why crystallographic/NMR studies of nucleic acids have proven difficult is because oligonucleotides often self-associate and/or equilibrate between several different conformations at the concentrations required for many high-resolution biophysical measurements. To help circumvent these problems, an array of chemical and biochemical approaches have been developed to explore the conformation dynamics of oligonucleotides. Perhaps the most common way to assess structure is by chemical and enzymatic footprinting. In footprinting, the accessibility and/or reactivity of particular functional groups in either a secondary or tertiary structure is examined and compared to the same positions in the denatured state. Footprinting experiments often employ base- and phosphate-specific modifying reagents, hydroxyl radical and transition metal complexes, and a broad spectrum of nucleases. Based on the cleavage properties of these reagents, as determined in control experiments, local structure can be studied. The most critical point to bear in mind when designing footprinting experiments is that since chemical and enzymatic structure probing is indirect, several different reagents should be employed to examine any given region of structure.

This chapter will provide the reader with a comprehensive collection of protocols to examine the solution structures of both DNA and RNA by footprinting. The initial units focus more heavily on methods to study RNA structure, while units appearing in future supplements will integrate more DNA protocols. UNIT 6.1 provides an extremely thorough coverage of the most useful chemical and enzymatic probes to examine RNA secondary and tertiary structure. For the chemical probes, both strand scission and primer extension detection protocols are provided.

Oftentimes, when RNA molecules adopt tertiary structure, distinct metal-binding pockets are created. Under appropriate conditions, this metal binding can be exploited to induce strand cleavage in the RNA backbone. This type of strand cleavage can be an exquisitely sensitive probe of local tertiary structure. UNITS 6.2-6.4 \& 6.8 describe six of the most useful metal complexes for probing nucleic acid structure.

UNITS $6.5 \& 6.7$ describe the application of hydroxyl radicals to investigate RNA and DNA tertiary structure, respectively. Unlike nearly every other footprinting reagent, hydroxyl radicals cleave the sugar-phosphate backbone at every residue. This reagent possesses little or no sequence selectivity and will provide uniform cleavage at all positions in a given RNA or DNA secondary structure. Because some positions often become protected from cleavage upon tertiary folding, this reagent is useful to monitor global folding at equilibrium. Moreover, as will be described in Chapter 11, hydroxyl radical cleavage can also be used to follow the kinetics of RNA folding.

Chemical and Enzymatic Probes for Nucleic Acid Structure

\subsection{1}

Supplement 13 
UNIT 6.6 presents a discussion of chemical reagents that can be used specifically to study the major groove of DNA. The unit focuses on the most commonly used and readily available reagents: dimethyl sulfate, diethylpyrocarbonate, potassium permanganate, osmium tetroxide, and bromine formed in situ from bromide and monoperoxysulfate. Characteristics of each reagent are reviewed, as are their applications to the study of DNA, DNA-protein complexes, and DNA-drug complexes.

Gary D. Glick

Introduction 